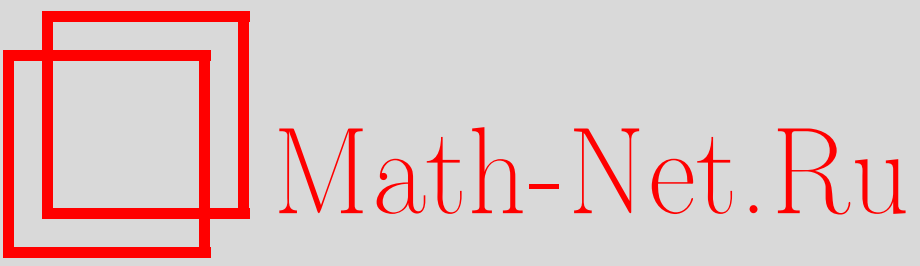

Ф. Бузато, Реализация универсальной формальной группы Абеля, УМH, 1996, том 51, выпуск 3, 183-184

DOI: https://doi.org/10.4213/rm975

Использование Общероссийского математического портала Math-Net.Ru подразумевает, что вы прочитали и согласны с пользовательским соглашением

http://www.mathnet.ru/rus/agreement

Параметры загрузки:

IP : 107.22 .136 .117

26 апреля 2023 г., 12:37:34 


\section{РЕАЛИЗАЦИЯ УНИВЕРСАЛЬНОЙ ФОРМАЛЬНОЙ ГРУППЫ АБЕЛЯ}

\section{Ф. БузАто}

Рассмотрим двухпараметрический род $\chi_{\alpha, \beta}: \Omega_{U} \rightarrow Q[\alpha, \beta]$, определяющий формальную группу с экспонентой

$$
\bar{g}(u)=\frac{1}{\alpha-\beta}(\exp (\alpha u)-\exp (\beta u)),
$$

где $\Omega_{U}$ - кольо комплексных кобордизмов. Эта формальная группа имеет вид

$$
f(u, v)=u+v+\alpha_{1} u v+\sum_{j>1} \alpha_{j}\left(u v^{j}+v u^{j}\right),
$$

она была названа в [2] именем $A 6 е л я$, который первый ввел функциональное уравнение (теорему сложения), характеризуюшее ряд $\bar{g}(u)$. Важными случаями рода $\chi_{\alpha, \beta}$ являются род Тодда $(\alpha=0, \beta=-1)$ и $A$-род $\left(\alpha=-\beta=\frac{1}{2}\right)$. В [2] вычислена универсальная формальная группа $A 6 е л я \mathscr{F}_{A b}(u, v)$. Основным результатом настоящей работы является

Теорема. Существует коммутативная комплексно-ориентируемая теория когомологий $A b^{*}(-)$ (теория кобордизмов с особенностями) с кольцом скаляров $A b^{*}$ (точка) $\cong \Lambda$, формальная группа $F_{A b}(u, v)$ в которой совпадает с $\mathscr{F}_{A b}(u, v)$, где $\Lambda$ - кольцо коэффициентов формальной группь $\mathscr{F}_{A b}(u, v)$.

Говоря о комплексно-ориентируемой теории когомологий, обычно предполагают, что она имеет коммутативное умножение. Однако, с точки зрения проблемы реализации формальных групп в теориях когомологий, достаточно использовать следующее

ОпрЕДЕлЕниЕ 1. Теория когомологий $h^{*}(-)$ называется комплексно-ориентируемой, если в ней определено функториальное, ассоциативное умножение, такое что

1) кольцо $h^{*}\left(\mathbb{C P}^{\infty} \times \mathbb{C P}^{\infty}\right)$ коммутативно;

2) вложение $i: S^{2} \simeq \mathbb{C P}^{1} \hookrightarrow \mathbb{C P}^{\infty}$ индуцирует эпиморфизм $i^{*}: h^{*}\left(\mathbb{C P}^{\infty}\right) \rightarrow h^{*}\left(S^{2}\right)$.

Для комплексно-ориентируемых теорий $h^{*}(-)$ характер Черна-Дольда $c h_{h}$ индуцирует кольевой изоморфизм

$$
\operatorname{ch}_{h} \otimes Q: h^{*}(X) \otimes Q \stackrel{\cong}{\longrightarrow} H^{*}\left(X ; h^{*}(\text { точка }) \otimes Q\right)
$$

для любого конечного клеточного комплекса $X$. Следовательно (как обратил мое внимание В. М. Бухштабер), коммутатор $[x, y]$ имеет конечный порядок для любых $x, y \in h^{*}(X)$.

Доказательство теоремы основывается на более общей конструкции, для которой фиксируем некоторые обозначения.

Пусть $R$ - коммутативное градуированное кольцо с единицей и $f(u, v)$ - одномерная коммутативная формальная группа над $R$. Через $F_{U}(u, v)=u+v+\sum_{i, j \geqslant 1} \alpha_{i, j} u^{i} v^{j}$ обозначим универсальную формальную группу над кольцом Лазара $L$ и через $\theta: L \rightarrow R$ - единственный кольцевой гомоморфизм из $L$ в $R$ такой, что $F(u, v)=\theta_{*} F_{U}(u, v)$.

ПрЕДЛОЖЕНИЕ 1. Если $R$ является областью челостности и $\theta$ является әпиморфизмом, то существует комплексно-ориентируемая теория когомологий $h^{*}(-)$, кольцо скаляров которой изоморфно $R$ и формальная группа которой равна $f(u, v)$, т.е. $h^{*}$ (точка) $\cong R$ u $F_{h}(u, v)=f(x, y)$.

Предложение 1 основывается на теориях (ко)бордизмов с особенностями [1] и, в частности, на работе О.К. Миронова [3], где доказано, что для произвольного множества особенностей $\Sigma$ в унитарной теории кобордизмов с особенностями $\Omega_{\Sigma}^{*}(-)$, существует ассоциативное допустимое умножение. Наложенные на $R$ и $\theta$ условия позволяют найти подходяший набор особенностей $\Sigma$, чтобы провести доказательство.

Работа выполнена при частичной поддержке Швейцарского Национального Фонда. 
ДокАЗАТЕЛЬСтво теОРемЫ. Часть первая. Напомним необходимые факты о $\Lambda$ - кольце

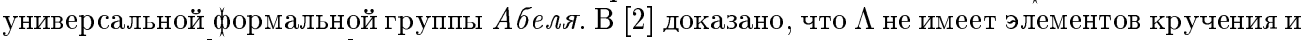
что $\Lambda \otimes Q=Q\left[\alpha_{1,1}, \alpha_{1,2}\right]$. Поэтому каноническое отображение $\Lambda \hookrightarrow \Lambda \otimes Q$ мономорфно, и, следовательно, $\Lambda$ является областью целостности. Кроме того, кольцо $\Lambda$ изоморфно фактор-кольцу $\Omega_{U}^{*} / J$, где $J$ - идеал, порожденный элементами $\alpha_{i, j}, i, j \geqslant 2$ (это следует из теоремы 3.4 в [2]). Итак, условия предложения 1 выполнены и первая часть теоремы доказана.

Остается исследовать коммутативность, так как предложение 1 дает не обязательно коммутативную теорию когомологий $h^{*}(-)$.

Препятствия к коммутативности можно вычислить с помощью операций Стинрода-Дика [3]. Коротко говоря, многообразие $[P]$ из множества особенностей $\Sigma$ определяет некоторый элемент $\kappa(P) \in \Omega_{\Sigma}^{*} \otimes Z_{2}$, являющийся препятствием к коммутативности в теории $\Omega_{\Sigma}^{*}(-)$. Обращаем внимание на то, что $\kappa(P)$ определено независимо от $\kappa(Q)$, если $[P] \neq[Q],[P],[Q] \in \Sigma$. Более того, выполнено $\kappa(P)=\widetilde{\mathscr{P}}_{2}([P])$, где отображение $\widetilde{\mathscr{P}}_{2}: \widetilde{\Omega}_{U}^{2 q}(X) \rightarrow \widetilde{\Omega}_{U}^{4 q}\left(\mathbb{R P}^{2} \wedge X\right)$ построено

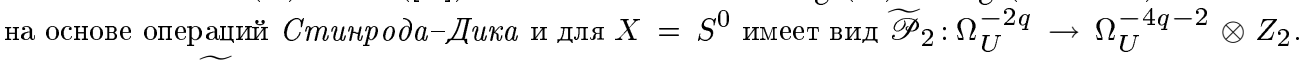
Отображение $\widetilde{\mathscr{P}}_{2}$ обладает следующими свойствами.

Лемма 1 [3]. 1) $\left.\widetilde{\mathscr{P}}_{2}(a \cdot b)=a^{2} \cdot \widetilde{\mathscr{P}}_{2}(b)+\widetilde{\mathscr{P}}_{2}(a) \cdot b^{2}, a, b \in \Omega_{U}^{*}(X) .2\right) \quad \widetilde{\mathscr{P}}_{2}(a+b)=$

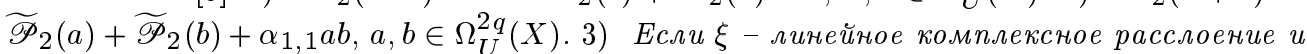
$u \in \Omega_{U}^{2}(T \xi)$ - его класс Тома, то имеет место формула

$$
\widetilde{\mathscr{P}}_{2}(u)=u+\sum_{k=1}^{\infty} \alpha_{1, k} s_{k}(u)=u+\sum_{k=1}^{\infty} \alpha_{1, k} u^{k+1},
$$

где $s_{k}$ - операчия Ландвебера-Новикова.

ДоКАЗАТЕЛЬСТво тЕОРЕМЫ. Часть вторая. С помощью леммы 1 и используя факт, что в колшце $\Lambda \cong \Omega_{U}^{*} / J$ верны соотношения

$$
k \alpha_{1, k}=-\sum_{n=2}^{k-1} n \alpha_{1, n} \alpha_{1, n-k}, \quad k \geqslant 3
$$

(см. [2]), проверяется, что $\widetilde{\mathscr{P}}_{2}(J) \equiv 0$ в $\Omega_{U}^{*} / J \otimes Z / 2$. Таким образом, все препятствия равны нулю, и, следовательно, теория $A b^{*}(-)$ является коммутативной. Теорема доказана.

В заключение приведем некоторые замечания о коммутативности в теориях $\Omega_{\Sigma}^{*}(-)$ для произвольного множества особенностей $\Sigma$.

Пусть $J_{\Sigma}$ - идеал в $\Omega_{U}^{*}$ (точка), порожденньй множеством $\Sigma$. Коммутативность умножения в теории $\Omega_{\Sigma}^{*}(-)$ эквивалентна тому, что $\widetilde{P}_{2}\left(J_{\Sigma}\right) \subset J_{\Sigma} \otimes Z / 2$. Итак, из тривиального представления любого элемента $x$ в виде $x=(2 k+1) x-2 k x$ следует (в [3] другим способом доказанная)

Лемма 2. Если $2 k+1 \in J_{\Sigma}(k \geqslant 1)$, то мультипликативная структура в $\Omega_{\Sigma}^{*}(-)$ является коммутативной.

На основе свойств отображении $\widetilde{\mathscr{P}}_{2}$ и теории формальных групп можно доказать следующий частный случай уже известной теоремы (см. [4] или [5]).

ПреДЛожение 2. Если $2 \in J_{\Sigma}$ и мультипликативная структура в $\Omega_{\Sigma}^{*}(-)$ коммутативна, то $\Omega_{\Sigma}^{*}(-) \cong H^{*}\left(-; \Omega_{\Sigma}^{*}(\right.$ точка $\left.)\right)$.

Автор благодарит В. М. Бухштабера за постановку задачи и полезные замечания.

\section{СПИСОК ЛИТЕРАТУРЫ}

[1] Baas N. A. // Math. Scand. 1973. V. 33. P. 279-302. [2] Бухштабер В. М., Холодов А. Н. // Матем. сб. 1990. Т. 181. № 1. С. 75-94. [3] Миронов О.К. // Изв. АН СССР. Сер. матем. 1978. Т. 42. № 4. С. 789-806. [4] Пажитнов А. В., Рудяк Ю. Б. // Матем. сб. 1984. T. 124 (166). № 4. C. 486-494. [5] Würgler U. // Comment. Math. Helvetici. 1986. V. 61. P. 33-45.

Московский государственный университет им. М.В. Ломоносова; Mathematisches Institut der Universität Bern, CH-3005 Bern
Принято редколлегией 18.05.1996 